\title{
Reactivity, Formation, and Solubility of Polyoxometalates Probed by Calorimetry
}

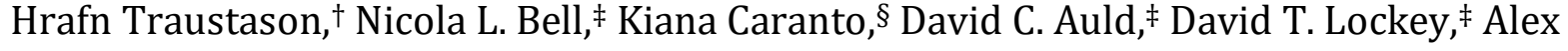 \\ Kokot,§ Jennifer E.S. Szymanowski,§ Leroy Cronin,‡ Peter C. Burns*,t,§ \\ †Department of Chemistry and Biochemistry, University of Notre Dame, Notre Dame, Indiana 46556, United \\ States \\ ‡School of Chemistry, University of Glasgow, University Avenue, Glasgow, G12 8QQ UK \\ § Department of Civil \& Environmental Engineering \& Earth Sciences, University of Notre Dame, Notre Dame, \\ Indiana 46556, United States
}

\section{Supporting Information}

\section{Table of Contents}

1. Synthesis

2. Uranyl Peroxide Clusters - Topology

3. Inductively Coupled Plasma - Optical Emission Spectrometry

4. Thermogravimetric Analysis

5. Electrospray Ionization - Mass Spectrometry

6. Calorimetric Measurements

7. Density Measurements

8. Thermographs

9. Calculated Values for $\Delta_{\mathrm{f}}{ }^{\circ} \mathrm{H}_{(\mathrm{aq})}$ Using $\Delta_{\mathrm{f}}{ }^{\circ} \mathrm{H}_{(\mathrm{c})}$ from Room Temperature Calorimetry 10. Thermochemical cycles

\section{Synthesis}

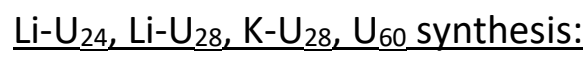

The syntheses for $\mathrm{Li}-\mathrm{U}_{24}, \mathrm{Li}-\mathrm{U}_{28}, \mathrm{~K}-\mathrm{U}_{28}$, and $\mathrm{U}_{60}$ were as previously published. ${ }^{1-3}$

Mo 132 Synthesis: 
An adapted literature procedure was used. ${ }^{4}$ Ammonium heptamolybdate tetrahydrate $(5.6 \mathrm{~g}$, $4.53 \mathrm{mmol}, 31.7 \mathrm{mmol}$ of $\mathrm{Mo}$ ) and ammonium acetate (12.5 g, $162.2 \mathrm{mmol})$ dissolved in $250 \mathrm{~mL}$ of water. Followed by the addition of hydrazine sulfate $(0.8 \mathrm{~g}, 6.15 \mathrm{mmol})$ and stirred for 10 minutes (colour change from colourless to blue-green). Then $83 \mathrm{ml}$ of $50 \%(\mathrm{v} / \mathrm{v}$ ) acetic acid is added. Stored in an open $500 \mathrm{~mL}$ conical flask without stirring (should colour slowly to brown). Red-brown crystals filtered off under vacuum after 4 days and washed with ethanol and diethyl ether.

Mo 154 Synthesis:

An adapted literature procedure was used. ${ }^{4}$ Sodium Molybdate dihydrate ( $\left.3.04 \mathrm{~g}, 12.56 \mathrm{mmol}\right)$ dissolved in $25 \mathrm{ml}$ of water and acidified with $2.7 \mathrm{~mL}$ of $32 \% \mathrm{HCl}$. This is followed by the addition of sodium dithionite $(0.15 \mathrm{~g}, 0.86 \mathrm{mmol}, 6.9 \mathrm{~mol} \%)$ under continuous stirring. Solution is kept in a closed flask for 1 day and crystals were filtered off and washed quickly with a very small amount of cold water and dried under vacuum.

\section{Mo Blue/Brown Interconversions}

Reduction of Mo 154 to Mo 132 :

To a stirred aqueous solution of $\mathrm{Mo}_{154}(0.99 \mathrm{~mL}, 6.6 \mathrm{mM}, 6.57 \mu \mathrm{mol})$ was added a solution of HOAC (5.45 mL, $4 \mathrm{M}, 21.8 \mathrm{mmol}),\left[\mathrm{N}_{2} \mathrm{H}_{5}\right]\left[\mathrm{HSO}_{4}\right](1 \mathrm{~mL}, 69 \mathrm{mM}, 69 \mu \mathrm{mol})$ and $\left[\mathrm{NH}_{4}\right] \mathrm{Cl}(1.44 \mathrm{~mL}, 2$ $\mathrm{M}, 2.88 \mathrm{mM}$ ) and the reaction mixture was diluted to $10 \mathrm{~mL}$ in a $14 \mathrm{~mL}$ vial. The solutions were stirred for $2 \mathrm{~h}$ before being stored at $18^{\circ} \mathrm{C}$ for 1 week after which the solutions were filtered and stored in open topped $14 \mathrm{~mL}$ vials at $18^{\circ} \mathrm{C}$ for 6 weeks. After this time brown needle-like crystals began to form which were characterized as the previously reported $\mathrm{Mo}_{134}$. Variations of this procedure by altering the $\left[\mathrm{NH}_{2} \mathrm{H}_{5}\right]\left[\mathrm{HSO}_{4}\right]$ and $\mathrm{HOAc}$ ratio by ca $20 \%$ gave only brown needles $\mathrm{Mo}_{134}$ (CSD: or white plate like crystals of $\mathrm{Mo}_{8}$ (in absence of ammonium chloride).

\section{Oxidation of Mo 132 to Mo 102 :}

To a stirred solution of $\mathrm{Mo}_{132}(1.94 \mathrm{~mL}, 2.1 \mathrm{mM}, 40.4 \mu \mathrm{mol})$ was added a solution of $\mathrm{H}_{2} \mathrm{O}_{2}(2.47$ $\mathrm{mL}, 29.4 \mathrm{mM}, 0.727 \mathrm{mmol})$ and $\mathrm{HCl}(202 \mu \mathrm{L}, 5 \mathrm{M}, 101 \mathrm{mmol})$ in a $14 \mathrm{~mL}$ vial. The solutions were stirred for $2 \mathrm{~h}$ before being caped and the cap pierced with a needle. The reaction vial was stored at $18^{\circ} \mathrm{C}$ for $48 \mathrm{~h}$ after which blue crystals began to form which were characterized as the previously reported $\mathrm{Mo}_{102}$. Variations of this procedure by altering the $\mathrm{H}_{2} \mathrm{O}_{2}$ and $\mathrm{HCl}$ ratio by ca $20 \%$ produced no evidence of $\mathrm{Mo}_{154}$. 


\section{Uranyl Peroxide Clusters - Topology}

Table S1. The topology of $\mathrm{U}_{24}, \mathrm{U}_{28}$, and $\mathrm{U}_{60}$ cage clusters broken down into topological windows.

\begin{tabular}{llll}
\hline$\underline{\text { Cluster }}$ & $\underline{\text { Square }}$ & $\underline{\text { Pentagonal }}$ & Hexagonal \\
\hline$\underline{U}_{24}$ & $\underline{6}$ & $\underline{0}$ & $\underline{8}$ \\
$\underline{U}_{28}$ & $\underline{0}$ & $\underline{12}$ & $\underline{4}$ \\
$\underline{U}_{60}$ & $\underline{0}$ & $\underline{12}$ & $\underline{20}$ \\
\hline
\end{tabular}

\section{Inductively Coupled Plasma - Optical Emission Spectrometry}

The elemental composition of the salt of each cluster was determined using a Perkin Elmer Optima 8000 DV-ICP-OES instrument. Three homogenous solutions were made by dissolving samples of clusters in $10 \mathrm{~mL} 5 \% \mathrm{HNO}_{3}$. The solutions were analyzed in triplicate, giving nine measurements in total. Yttrium was added to all samples and standards as an internal standard at concentrations of $0.5 \mathrm{ppm}$ and $1.5 \mathrm{ppm}$, respectively. Ten external standards were used for calibration with concentrations of Li ranging from 0.02 to $1.1 \mathrm{ppm}, \mathrm{K}$ ranging from 0.9 to $5.6, \mathrm{U}$ ranging from 3.2 to $34.6 \mathrm{ppm}$, Na from 0.01 to 10.2, and Mo ranging from 1.1 to 35.3. Samples with concentration values outside the calibration were excluded from the measurement. Error is represented as one standard deviation.

Table S2. Ratio of elements obtained from ICP-OES results.

\begin{tabular}{cccc}
\hline Compound & Li:U ratio & K:U ratio & Mo:Na ratio \\
\hline Li U24 & $1.02(4)$ & - & - \\
Li U28 & $1.01(5)$ & - & - \\
K U28 & - & $1.03(7)$ & - \\
U60 & $0.66(4)$ & $0.34(3)$ & - \\
Mo132 & - & - & $0.096(11)$ \\
Mo154 & - & - & \\
\hline
\end{tabular}

\section{Thermogravimetric Analysis}

Thermogravimetric analyses were conducted for aliquots of each POM using a Mettler-Todelo TGA-DSC 1. For each measurement, crystals were placed in an Al crucible and heated to 600$750^{\circ} \mathrm{C}$ at a rate of $5^{\circ} \mathrm{C} / \mathrm{min}$ under $\mathrm{Ar}$ gas flowing at $50 \mathrm{~mL} / \mathrm{min}$. 
For analysis, mass loss by $450^{\circ} \mathrm{C}$ was used for uranyl peroxide clusters and $200^{\circ} \mathrm{C}$ for Mo POMs.
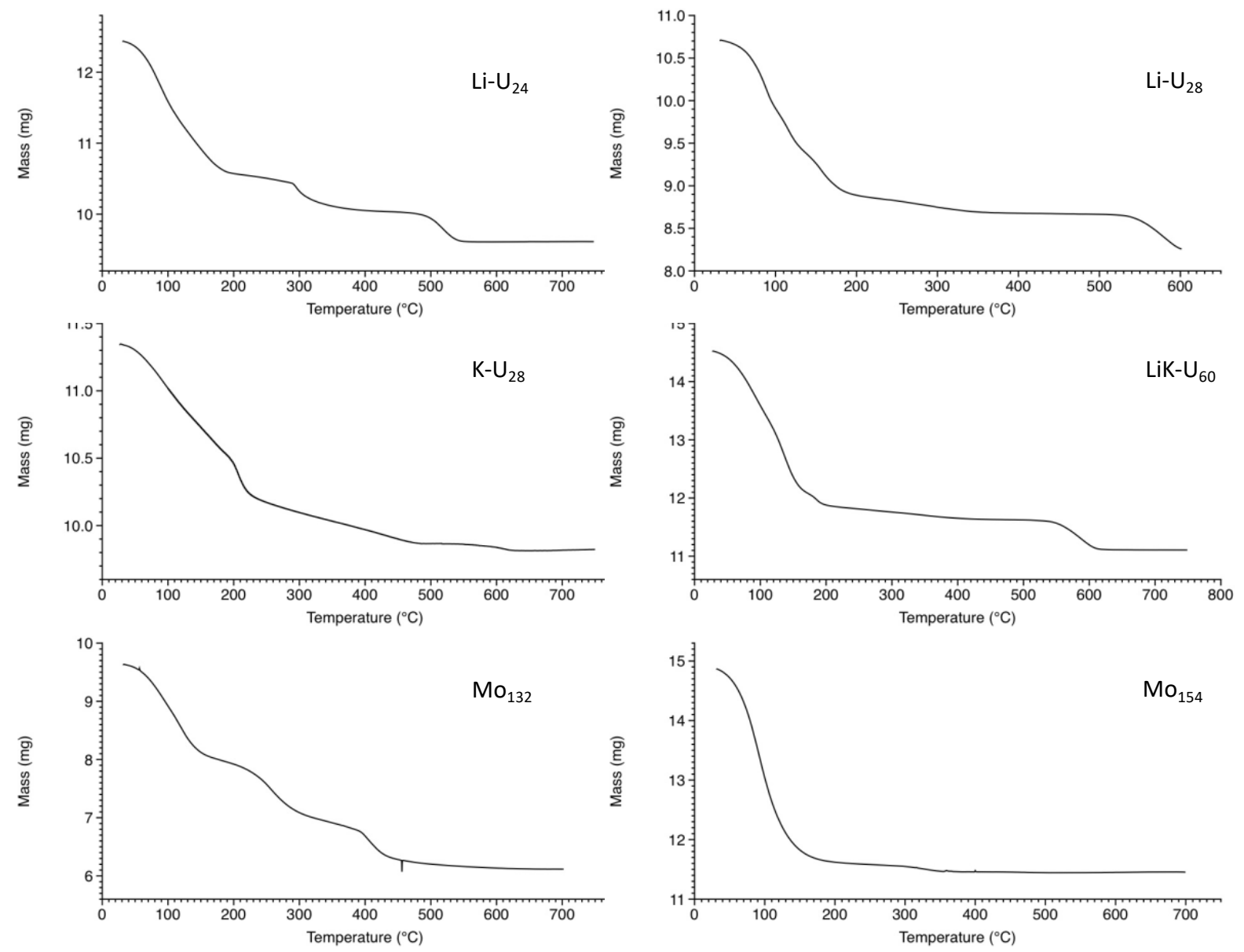

Figure S1. Thermogravimetric curves of $\mathrm{Li}-\mathrm{U}_{24}, \mathrm{Li}-\mathrm{U}_{28}, \mathrm{~K}-\mathrm{U}_{28}, \mathrm{LiK}-\mathrm{U}_{60}, \mathrm{Mo}_{132}$, and $\mathrm{Mo}_{154}$.

\section{Electrospray Ionization - Mass Spectrometry}

Electrospray ionization mass spectra were collected using a Bruker micrOTOF-Q II highresolution quadropole time-of-flight spectrometer in negative ion mode with a $2900 \mathrm{~V}$ capillary voltage, 0.85 bar of nebulizer, and $4 \mathrm{~L} / \mathrm{min}$ of dry gas. Uranium containing samples were diluted to less than $2000 \mathrm{ppm}$ of $U$ in water immediately prior to introduction into the system by direct injection. Data were collected over 3 min with an injection rate of $600 \mu \mathrm{L} / \mathrm{h}$ and scanned over the $500-5000 \mathrm{~m} / \mathrm{z}$ region. Charge states were assigned to the resulting peaks using the Bruker software DataCompass. 

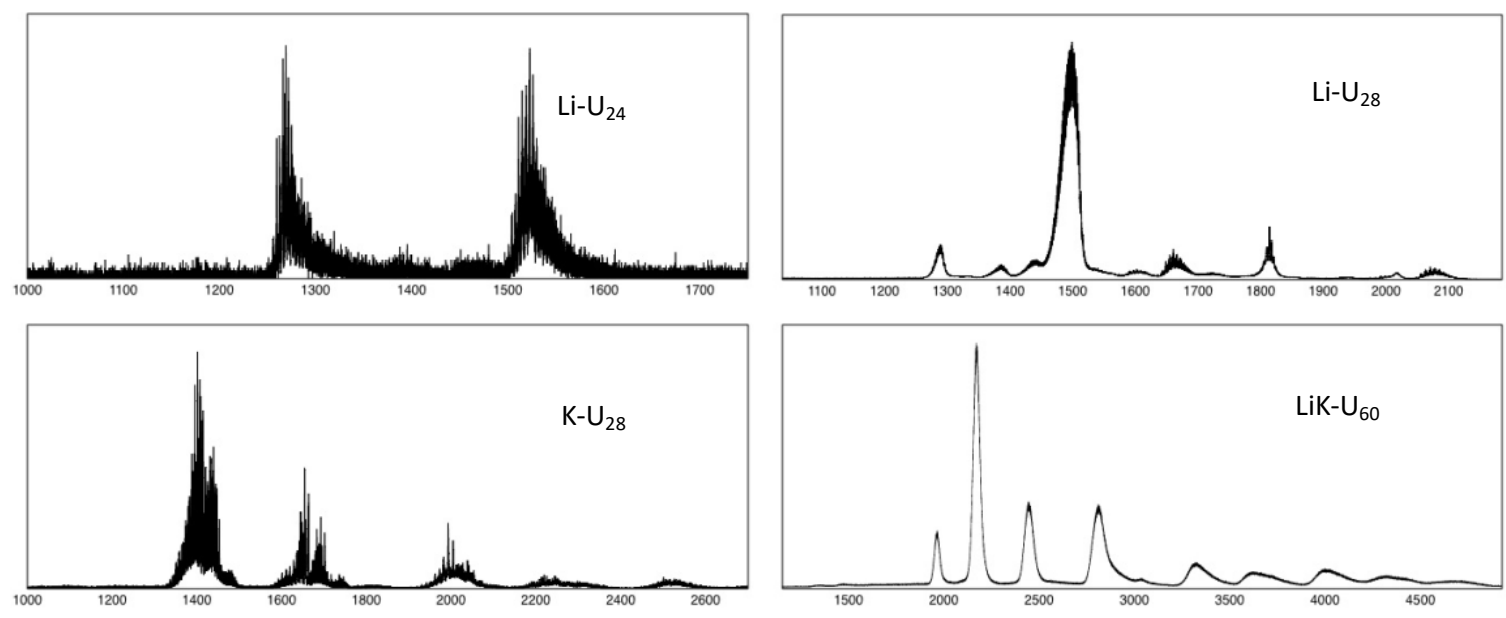

Figure S2. ESI-MS spectra of $\mathrm{Li}_{-} \mathrm{U}_{24}, \mathrm{Li}-\mathrm{U}_{28}, \mathrm{~K}-\mathrm{U}_{28}$, and $\mathrm{LiK}-\mathrm{U}_{60}$.

\section{Calorimetric Measurements}

The standard equipment and procedures provided by Setaram for the C-80 were modified because of the radioactivity of uranium and the reactivity of reactants to stainless steel. Polyether ether ketone (PEEK) sample holders were designed to replace the stainless-steel holders from Setaram. For UPC measurements $6 \mathrm{M} \mathrm{HCl}$ was used to destroy the clusters. Polypropylene (PP) tubes holding reactant $B$ (acid or water) were placed in the PEEK sample holders, and a borosilicate vial containing reactant $A$ (the sample) was carefully placed inside the PP tube so that reactant $A$ and $B$ do not mix. The PEEK sample holder was next placed inside the calorimeter sample chamber. The same set-up was placed in the reference chamber but without reactant $A$. The sample and reference chambers were locked as a closed system and allowed to stabilize until thermal equilibrium had been established. Once thermal equilibrium had been attained, the mixing mechanism of the instrument was turned on and reactants $A$ and $B$ mixed.

In the case of Mo based POMs, $4 \mathrm{M} \mathrm{NaOH}$ was used to break the POMs down into simple species. Due to the reactivity between borosilicate and strong base, the base was placed into PEEK sample holders and the sample was placed in a PP tube before being inserted into the calorimeter chamber. 
a)

b)

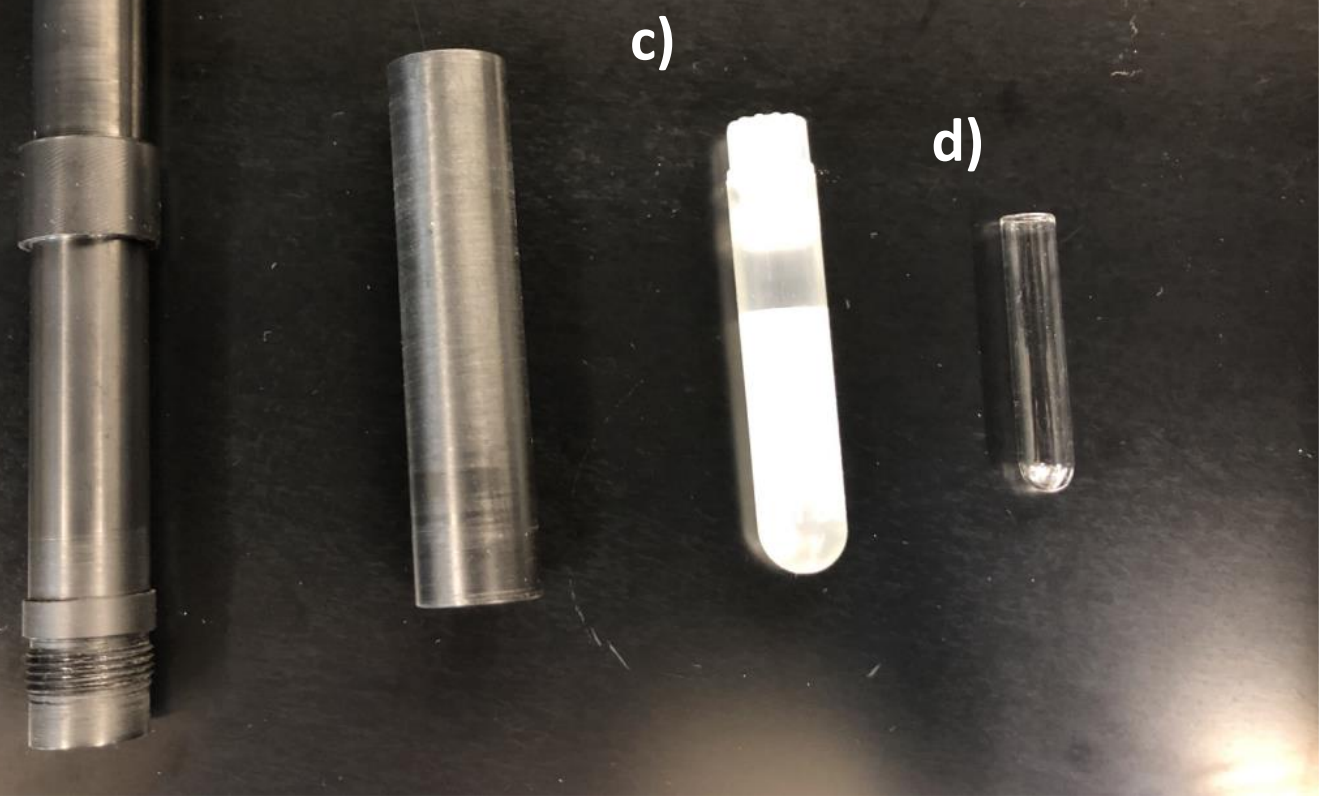

Figure S3. a) PEEK sample holder, top part, b) PEEK sample holder, bottom part, c) polypropylene tube, and d) borosilicate vial.

The thermochemical cycles adhere to Hess's law that states that enthalpy is a function of state and therefore is the standard enthalpy of formation of a compound equal to the sum of enthalpies of reactions as reactions sum up to the equivalence of the overall reaction. As these experiments were the first of their kind, it was necessary to measure the enthalpies of the additional compounds used in the thermochemical cycles.

Calorimetric measurements involving aqueous POMs were more challenging than the

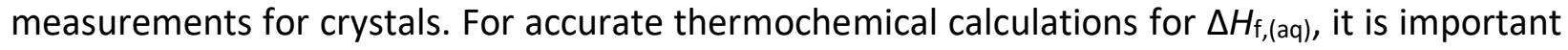
to maintain consistent concentrations for all compounds measured for thermochemical calculations with respect to the aqueous POM. It was also important for all POM solutions to be the same concentration (0.5-2 $\mathrm{mM}$ ) regardless of which POM is being measured. Due to the low concentration of POMs and additional compounds needed for thermochemical cycles (0.5-2 $\mathrm{mM})$, the heat of reaction for the simpler compounds $\left(\mathrm{UO}_{2}\left(\mathrm{NO}_{3}\right)_{2}, \mathrm{LiOH}\right.$, etc.) was overwhelmed by the heat of dilution of the acid or base. Hence, the methodology was adjusted.

The heat of reaction for $\mathrm{H}_{2} \mathrm{O}+6 \mathrm{M} \mathrm{HCl}$ and $\mathrm{H}_{2} \mathrm{O}+4 \mathrm{M} \mathrm{NaOH}$ was measured separately and the value subtracted from the heat measured for all samples. Stock solutions for each nonPOM compound needed for thermochemical calculations were made. The stock was 
gravimetrically diluted in series into 4-6 solutions, the density of each diluted solution was measured, and the concentration for each solution calculated using Equation S1. The mass of reactant $A$ and $B$ (sample and acid/base) were recorded prior to being loaded into the calorimeter and calorimetric measurements were repeated eight times for each solution. The enthalpy of reaction was calculated using Equation S2. Next, the enthalpy of reaction was plotted graphically with respect to concentration and determined by extrapolation to the appropriate concentration for each thermochemical cycle.

$$
\begin{aligned}
& M=\frac{\text { Mass } \% \times \rho}{\text { Molas Mass }} \\
& \Delta H_{r x n}= \frac{\left(Q_{r x n}-Q_{\mathrm{H}_{2} \mathrm{O}}\right) \times \rho}{M \times m} \times m \\
& \text { Equation } \mathbf{~ 2 2}
\end{aligned}
$$

\section{Density Measurements}

Density measurements were performed using Anton Paar DMA 3400 M density meter.

Table S3. Results from density measurements of standards used in thermochemical calculations.

\begin{tabular}{lllll}
\hline Compound & $\begin{array}{l}\text { UO2(NO3)2 } \\
\text { Density (g/L) }\end{array}$ & $\begin{array}{l}\text { LiOH } \\
\text { Density (g/L) }\end{array}$ & $\begin{array}{l}\text { KOH } \\
\text { Density (g/L) }\end{array}$ & $\begin{array}{l}\text { CH3CO2H } \\
\text { Density (g/L) }\end{array}$ \\
\hline Solution \#1 & 1.27220 & 1.02367 & 1.04046 & 1.00697 \\
Solution \#2 & 1.20432 & 1.01751 & 1.03038 & 1.00527 \\
Solution \#3 & 1.06701 & 1.01132 & 1.02091 & 1.00349 \\
Solution \#4 & 1.03279 & 1.00492 & 1.00983 & 1.00182 \\
Solution \#5 & $\mathrm{n} / \mathrm{a}$ & 0.99954 & $\mathrm{n} / \mathrm{a}$ & 1.00192 \\
\hline
\end{tabular}

Table S4. Results from density measurements of cluster solutions.

\begin{tabular}{ll}
\hline Compound & Density $(\mathrm{g} / \mathrm{L})$ \\
\hline Li U24 & 1.00611 \\
Li U28 & 1.00901 \\
K U28 & 1.01117 \\
U60 & 1.01494 \\
Mo132 & 1.02170 \\
Mo154 & 1.01676 \\
\hline
\end{tabular}

\section{Thermographs}



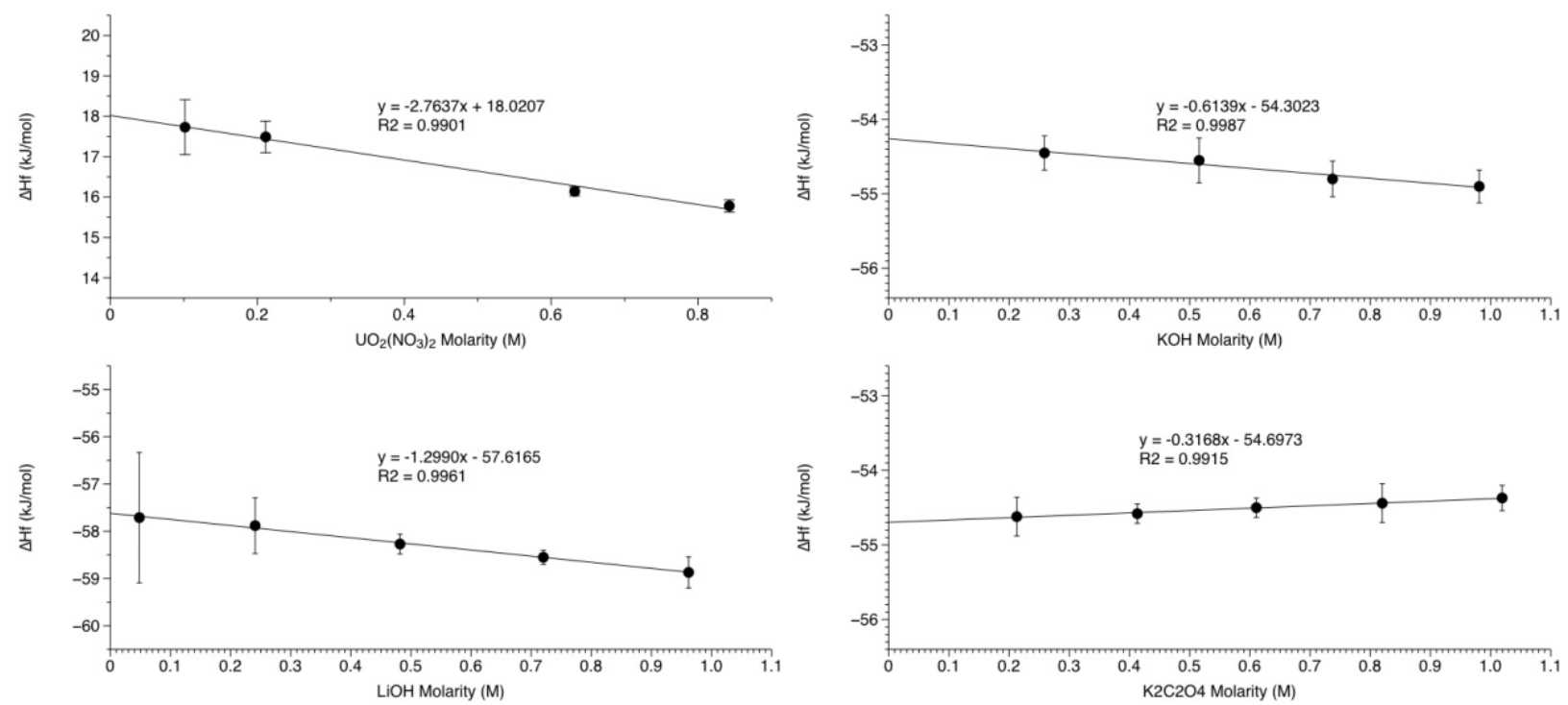

Figure S4. Thermographs for $\mathrm{UO}_{2}\left(\mathrm{NO}_{3}\right)_{2(\mathrm{aq})}, \mathrm{KOH}_{(\mathrm{aq})}, \mathrm{LiOH}_{(\mathrm{aq})}$, and $\mathrm{K}_{2} \mathrm{C}_{2} \mathrm{O}_{4(\mathrm{aq})}$ used to extrapolate to the appropriate $\Delta H$ value for thermochemical calculations.

\section{Calculated Values for $\Delta_{f}{ }^{\circ} H_{(a q)}$ Using $\Delta_{f}{ }^{\circ} H_{(c)}$ from Room Temperature Calorimetry}

Table S5. The calculated values for $\Delta_{f}{ }^{\circ} H_{(a q)}$ using $\Delta_{f}{ }^{\circ} H_{(c)}$ from room temperature calorimetry are in worse agreement with the measured values indicating that the high temperature calorimetry method provides better accuracy for $\Delta_{f}{ }^{\circ} H_{(c)}$.

\begin{tabular}{llll}
\hline POM & $\Delta H_{\mathrm{f}}{ }^{\circ}{ }_{\text {aq }} / M-$ Calculated $(\mathrm{kJ} / \mathrm{mol}$ & $\left.\Delta H_{\mathrm{f}}{ }^{\circ}{ }_{\mathrm{aq}}\right) / M-$ Measured $(\mathrm{kJ} / \mathrm{mol})$ & Discrepancy \\
\hline $\mathrm{Li}_{-} \mathrm{U}_{24}$ & $-1,534.6 \pm 11.0$ & $-1,837.0 \pm 0.4$ & $9.74 \%$ \\
$\mathrm{Li}_{-} \mathrm{U}_{28}$ & $-1,534.6 \pm 0.7$ & $-1694.0 \pm 0.7$ & $9.38 \%$ \\
$\mathrm{~K}-\mathrm{U}_{28}$ & $-1,405.6 \pm 14.5$ & $-1,670.7 \pm 0.1$ & $15.87 \%$ \\
$\mathrm{U}_{60}$ & $-1,695.8 \pm 0.6$ & $-1,837.6 \pm 0.5$ & $7.72 \%$ \\
$\mathrm{Mo}_{132}$ & $-1,041.4 \pm 3.9$ & $-1,040.1 \pm 10.8$ & $0.13 \%$ \\
\hline
\end{tabular}

\section{Thermochemical Cycles}

Table S6. Error is represented as two standard deviations from the mean. $\mathrm{n}=$ excess, $M=$ molarity.

\begin{tabular}{|c|c|c|c|}
\hline Reaction & & & $\Delta H(\mathrm{~kJ} / \mathrm{mol})$ \\
\hline $\begin{array}{l}\mathrm{Li}_{24}\left[\mathrm{UO}_{2} \mathrm{O}_{2} \mathrm{OH}\right]_{24} \cdot 83 \mathrm{H}_{2} \mathrm{O}_{(\mathrm{c})}+ \\
\mathrm{Li}_{24}\left[\mathrm{UO}_{2} \mathrm{O}_{2} \mathrm{OH}\right]_{24(\mathrm{aq})}+(\mathrm{n}+83) \mathrm{H}_{2} \mathrm{O}_{(\mathrm{l})}\end{array}$ & $\mathrm{n}$ & $\mathrm{H}_{2} \mathrm{O}_{(I)}=$ & $\Delta H_{1}=\Delta H_{\text {diss }}=82.2 \pm 13.5$ \\
\hline
\end{tabular}




\begin{tabular}{|c|c|}
\hline $\begin{array}{l}\mathrm{Li}_{28}\left[\mathrm{UO}_{2}\left(\mathrm{O}_{2}\right)_{1.5}\right]_{28} \cdot 73 \mathrm{H}_{2} \mathrm{O}_{(\mathrm{c})}+\mathrm{n}+\mathrm{H}_{2} \mathrm{O}_{(\mathrm{l})}= \\
\mathrm{Li}_{28}\left[\mathrm{UO}_{2}\left(\mathrm{O}_{2}\right)_{1.5}\right]_{28(\mathrm{aq})}+(\mathrm{n}+73) \mathrm{H}_{2} \mathrm{O}_{(\mathrm{l})}\end{array}$ & $\Delta H_{2}=\Delta H_{\text {diss }}=9.8 \pm 1.4$ \\
\hline $\begin{array}{l}\mathrm{K}_{28}\left[\mathrm{UO}_{2}\left(\mathrm{O}_{2}\right)_{1.5}\right]_{28} \cdot 43.5 \mathrm{H}_{2} \mathrm{O}_{(\mathrm{c})}+\mathrm{n} \mathrm{H}_{2} \mathrm{O}_{(\mathrm{l})}= \\
\mathrm{K}_{28}\left[\mathrm{UO}_{2}\left(\mathrm{O}_{2}\right)_{1.5}\right]_{28(\mathrm{aq})}+(\mathrm{n}+43.5) \mathrm{H}_{2} \mathrm{O}_{(\mathrm{l})}\end{array}$ & $\Delta H_{3}=\Delta H_{\text {diss }}=145.86 \pm 21.0$ \\
\hline $\begin{array}{l}\mathrm{Li}_{40} \mathrm{~K}_{20}\left[\mathrm{UO}_{2} \mathrm{O}_{2} \mathrm{OH}\right]_{60} \cdot 227 \mathrm{H}_{2} \mathrm{O}_{(\mathrm{c})}+\mathrm{n} \mathrm{H}_{2} \mathrm{O}_{(\mathrm{l})}= \\
\mathrm{Li}_{40} \mathrm{~K}_{20}\left[\mathrm{UO}_{2} \mathrm{O}_{2} \mathrm{OH}\right]_{60(\mathrm{aq})}+(\mathrm{n}+227) \mathrm{H}_{2} \mathrm{O}_{(\mathrm{l})}\end{array}$ & $\Delta H_{4}=\Delta H_{\text {diss }}=182.97 \pm 2.5$ \\
\hline 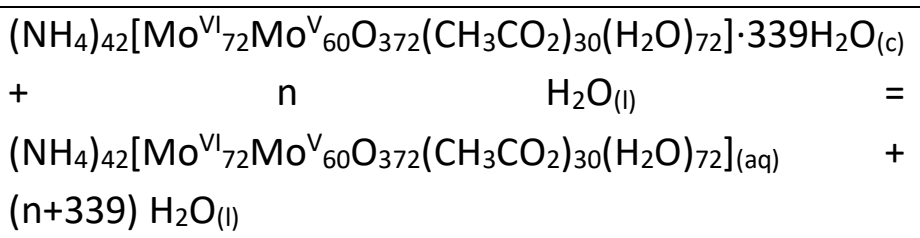 & $\Delta H_{5}=\Delta H_{\text {diss }}=146.2 \pm 18.8$ \\
\hline $\begin{array}{l}\mathrm{Na}_{15}\left[\mathrm{MoVI}_{126} \mathrm{Mo}_{28} \mathrm{O}_{462} \mathrm{H}_{14}\left(\mathrm{H}_{2} \mathrm{O}\right)_{70}\right]_{0.5} \\
{\left[\mathrm{MoVI}_{124} \mathrm{MoV}_{28} \mathrm{O}_{457} \mathrm{H}_{14}\left(\mathrm{H}_{2} \mathrm{O}\right)_{68}\right]_{0.5} \cdot 384 \mathrm{H}_{2} \mathrm{O}_{(\mathrm{c})}+\mathrm{n} \mathrm{H}_{2} \mathrm{O}_{(\mathrm{l})}=} \\
\mathrm{Na}_{15}\left[\mathrm{MoVI}_{126} \mathrm{Mo}_{28} \mathrm{O}_{462} \mathrm{H}_{14}\left(\mathrm{H}_{2} \mathrm{O}\right)_{70}\right]_{0.5} \\
{\left[\mathrm{MoVI}_{124} \mathrm{Mov}_{28} \mathrm{O}_{457} \mathrm{H}_{14}\left(\mathrm{H}_{2} \mathrm{O}\right)_{68}\right]_{0.5(\mathrm{aq})}+(\mathrm{n}+384) \mathrm{H}_{2} \mathrm{O}_{(\mathrm{l})}}\end{array}$ & $\Delta H_{6}=\Delta H_{\text {diss }}=146.5 \pm 8.2$ \\
\hline $\begin{array}{l}\mathrm{Li}_{24}\left[\mathrm{UO}_{2} \mathrm{O}_{2} \mathrm{OH}\right]_{24(\mathrm{aq})}+72 \mathrm{HCl}_{(\mathrm{aq})}=24 \mathrm{Li}^{+}{ }_{\text {(aq, dil) }}+24 \\
\mathrm{UO}_{2}{ }^{2+}{ }_{\text {(aq, dil) }}+48 \mathrm{H}_{2} \mathrm{O}_{(\mathrm{l})}+12 \mathrm{O}_{2(\mathrm{~g})}+72 \mathrm{Cl}^{-}{ }_{\text {(aq, dil) }}\end{array}$ & $\Delta H_{7}=-297.2 \pm 18.0$ \\
\hline $\begin{array}{l}\mathrm{Li}_{28}\left[\mathrm{UO}_{2}\left(\mathrm{O}_{2}\right)_{1.5}\right]_{28(\mathrm{aq})}+84 \mathrm{HCl}_{(\mathrm{aq})}=28 \mathrm{Li}^{+}{ }_{(\mathrm{aq}, \text { dil })}+28 \\
\mathrm{UO}_{2}{ }^{2+}{ }_{\text {(aq, dil) }}+42 \mathrm{H}_{2} \mathrm{O}_{(\mathrm{l})}+21 \mathrm{O}_{2(\mathrm{~g})}+84 \mathrm{Cl}^{-}{ }_{\text {(aq, dil }}\end{array}$ & $\Delta H_{8}=-350.9 \pm 23.7$ \\
\hline $\begin{array}{l}\mathrm{K}_{28}\left[\mathrm{UO}_{2}\left(\mathrm{O}_{2}\right)_{1.5}\right]_{28(\mathrm{aq})}+84 \mathrm{HCl}_{(\mathrm{aq})}=28 \mathrm{~K}_{(\mathrm{aq}, \mathrm{dil})}+\mathrm{UO}_{2}{ }^{2+}{ }_{(\mathrm{aq}} \\
\text { dil) }+42 \mathrm{H}_{2} \mathrm{O}_{(\mathrm{l})}+21 \mathrm{O}_{2(\mathrm{~g})}+84 \mathrm{Cl}_{(\mathrm{aq}, \mathrm{dil})}^{-}\end{array}$ & $\Delta H_{9}=-174.3 \pm 12.0$ \\
\hline $\mathrm{Li}_{40} \mathrm{~K}_{20}\left[\mathrm{UO}_{2} \mathrm{O}_{2} \mathrm{OH}\right]_{60(\mathrm{aq})}+180 \mathrm{HCl}_{(\mathrm{aq})}=40 \mathrm{Li}_{(\mathrm{aq}, \mathrm{dil})}+20$ & \\
\hline $\begin{array}{l}\mathrm{K}^{+}{ }_{(\mathrm{aq}, \text { dil })}+60 \mathrm{UO}_{2}{ }^{2+}{ }_{(\mathrm{aq}, \text { dil })}+120 \mathrm{H}_{2} \mathrm{O}_{(\mathrm{l})}+30 \mathrm{O}_{2(\mathrm{~g})}+180 \mathrm{Cl}^{-} \\
\text {(aq, dil) }\end{array}$ & $\Delta H_{10}=-73.5 \pm 17.8$ \\
\hline 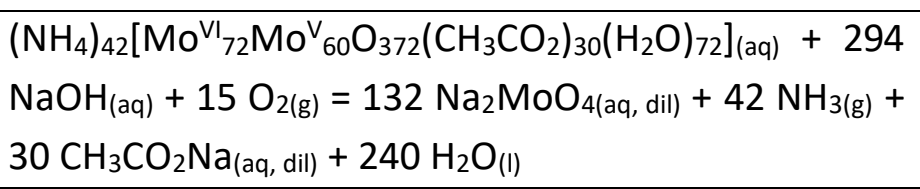 & $\Delta H_{11}=-13,243.5 \pm 649.3$ \\
\hline $\begin{array}{l}\mathrm{Na}_{15}\left[\mathrm{MoVI}_{126} \mathrm{Mo}_{28} \mathrm{~V}_{462} \mathrm{H}_{14}\left(\mathrm{H}_{2} \mathrm{O}\right)_{70}\right]_{0.5} \\
{\left[\mathrm{Mo}_{124} \mathrm{MoV}_{28} \mathrm{O}_{457} \mathrm{H}_{14}\left(\mathrm{H}_{2} \mathrm{O}\right)_{68}\right]_{0.5(\mathrm{aq})}+291 \mathrm{NaOH}_{(\mathrm{aq})}+7} \\
\mathrm{O}_{2(\mathrm{~g})}=153 \mathrm{Na}_{2} \mathrm{MoO}_{(\text {aq, dil) }}+221.5 \mathrm{H}_{2} \mathrm{O}_{(\mathrm{l})}\end{array}$ & $\Delta H_{12}=-12,268.6 \pm 65.7$ \\
\hline $\begin{array}{l}\mathrm{Li}_{24}\left[\mathrm{UO}_{2} \mathrm{O}_{2} \mathrm{OH}\right]_{24} \cdot 83 \mathrm{H}_{2} \mathrm{O}_{(\mathrm{c})}+72 \mathrm{HCl}_{(\mathrm{aq})}=24 \mathrm{Li}^{+}{ }_{(\mathrm{aq})}+24 \\
\mathrm{UO}_{2}{ }^{2+}{ }_{\text {(aq) }}+131 \mathrm{H}_{2} \mathrm{O}_{(\mathrm{l})}+12 \mathrm{O}_{2(\mathrm{~g})}+72 \mathrm{Cl}_{(\mathrm{aq})}^{-}\end{array}$ & $\Delta H_{13}=-4,217.3 \pm 264.6$ \\
\hline
\end{tabular}




\begin{tabular}{|c|c|}
\hline $\begin{array}{l}\mathrm{Li}_{28}\left[\mathrm{UO}_{2}\left(\mathrm{O}_{2}\right)_{1.5}\right]_{28} \cdot 73 \mathrm{H}_{2} \mathrm{O}_{(\mathrm{c})}+84 \mathrm{HCl}_{(\mathrm{aq})}=28 \mathrm{Li}^{+}{ }_{(\mathrm{aq})}+28 \\
\mathrm{UO}_{2}{ }^{2+}{ }_{(\mathrm{aq})}+115 \mathrm{H}_{2} \mathrm{O}_{(\mathrm{l})}+21 \mathrm{O}_{2(\mathrm{~g})}+84 \mathrm{Cl}_{(\mathrm{aq})}\end{array}$ & $\Delta H_{14}=-4,447 \pm 135.1$ \\
\hline $\begin{array}{l}\mathrm{K}_{28}\left[\mathrm{UO}_{2}\left(\mathrm{O}_{2}\right)_{1.5}\right]_{28} \cdot 43.5 \mathrm{H}_{2} \mathrm{O}_{(\mathrm{c})}+84 \mathrm{HCl}_{(\mathrm{aq})}=28 \mathrm{~K}^{+}{ }_{(\mathrm{aq})}+28 \\
\mathrm{UO}_{2}{ }^{2+}{ }_{(\mathrm{aq})}+85.5 \mathrm{H}_{2} \mathrm{O}_{(\mathrm{l})}+21 \mathrm{O}_{2(\mathrm{~g})}+84 \mathrm{Cl}^{-}{ }_{(\mathrm{aq})}\end{array}$ & $\Delta H_{15}=-5,704.5 \pm 406.8$ \\
\hline $\begin{array}{l}\mathrm{Li}_{40} \mathrm{~K}_{20}\left[\mathrm{UO}_{2} \mathrm{O}_{2} \mathrm{OH}\right]_{60} \cdot 227 \mathrm{H}_{2} \mathrm{O}_{(\mathrm{c})}+180 \mathrm{HCl}_{(\mathrm{aq})}=40 \mathrm{Li}^{+}{ }_{(\mathrm{aq})} \\
+20 \mathrm{~K}_{(\mathrm{aq})}+60 \mathrm{UO}_{2}{ }^{2+}{ }_{(\mathrm{aq})}+347 \mathrm{H}_{2} \mathrm{O}_{(\mathrm{l})}+30 \mathrm{O}_{2(\mathrm{~g})}+180 \mathrm{Cl}^{-} \\
\text {(aq) }\end{array}$ & $\Delta H_{16}=-6,684.1 \pm 30.9$ \\
\hline 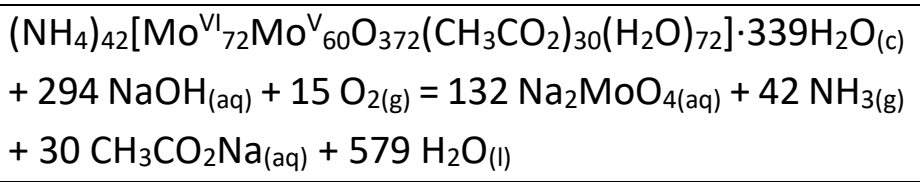 & $\Delta H_{17}=-12,923.3 \pm 519.8$ \\
\hline $\begin{array}{l}\mathrm{Li}_{24}\left[\mathrm{UO}_{2} \mathrm{O}_{2} \mathrm{OH}\right]_{24} \cdot 83 \mathrm{H}_{2} \mathrm{O}_{(\mathrm{c})}=12 \mathrm{Li}_{2} \mathrm{O}_{\left(\mathrm{sln}, 700^{\circ} \mathrm{C}\right)}+24 \\
\mathrm{UO}_{3\left(\mathrm{~s} \ln , 700^{\circ} \mathrm{C}\right)}+12 \mathrm{O}_{2\left(\mathrm{~g}, 700^{\circ} \mathrm{C}\right)}+95 \mathrm{H}_{2} \mathrm{O}_{\left(\mathrm{g}, 700^{\circ} \mathrm{C}\right)}\end{array}$ & $\Delta H_{18}=\Delta H_{\mathrm{ds}}=8,586.6 \pm 315.0$ \\
\hline $\begin{array}{l}\mathrm{Na}_{15}\left[\mathrm{MoVI}_{126} \mathrm{Mo}_{28}{ }_{28} \mathrm{O}_{462} \mathrm{H}_{14}\left(\mathrm{H}_{2} \mathrm{O}\right)_{70}\right]_{0.5} \\
{\left[\mathrm{Mo}{ }_{124} \mathrm{MoV}_{28} \mathrm{O}_{457} \mathrm{H}_{14}\left(\mathrm{H}_{2} \mathrm{O}\right)_{68}\right]_{0.5} \cdot 384 \mathrm{H}_{2} \mathrm{O}_{(\mathrm{c})}+7 \mathrm{O}_{2(\mathrm{~g},}} \\
\left.\left.700^{\circ} \mathrm{C}\right)=7.5 \mathrm{Na}_{2} \mathrm{O}_{(\mathrm{s})}, 700^{\circ} \mathrm{C}\right)+153 \mathrm{MoO}_{3\left(\mathrm{sln}, 700^{\circ} \mathrm{C}\right)}+460 \\
\mathrm{H}_{2} \mathrm{O}_{\left(\mathrm{g}, 700^{\circ} \mathrm{C}\right)}\end{array}$ & $\Delta H_{19}=\Delta H_{\mathrm{ds}}=30,582.3 \pm 4343.0$ \\
\hline 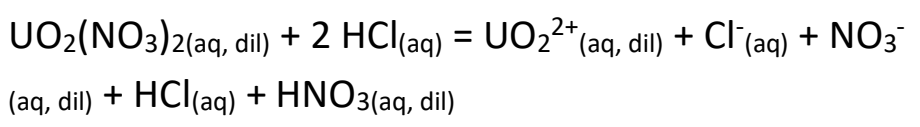 & $\Delta H_{20}=15.3 \pm 1.3$ \\
\hline $\mathrm{LiOH}_{(\mathrm{aq}, \text { dil) }}+\mathrm{HCl}_{(\mathrm{aq})}=\mathrm{Li}^{+}{ }_{(\mathrm{aq}, \text { dil) }}+\mathrm{Cl}^{-}(\mathrm{aq}$, dil) $)+\mathrm{H}_{2} \mathrm{O}_{(\mathrm{l})}$ & $\Delta H_{21}=-58.9 \pm 2.6$ \\
\hline $\mathrm{KOH}_{(\mathrm{aq}, \mathrm{dil})}+\mathrm{HCl}_{(\mathrm{aq})}=\mathrm{K}_{(\mathrm{aq}, \mathrm{dil})}+\mathrm{Cl}_{(\mathrm{aq}, \mathrm{dil})}^{-}+\mathrm{H}_{2} \mathrm{O}_{(\mathrm{l})}$ & $\Delta H_{22}=-54.9 \pm 1.4$ \\
\hline $\mathrm{CH}_{3} \mathrm{CO}_{2} \mathrm{H}_{(\text {(aq, dil) }}+\mathrm{NaOH}_{(\mathrm{aq})}=\mathrm{CH}_{3} \mathrm{CO}_{2} \mathrm{Na}$ (aq, dil) $+\mathrm{H}_{2} \mathrm{O}_{(\mathrm{l})}$ & $\Delta H_{23}=-54.7 \pm 3.6$ \\
\hline $\mathrm{UO}_{2}\left(\mathrm{NO}_{3}\right)_{2(\mathrm{aq}, 1 \mathrm{M})}=\mathrm{UO}_{2}\left(\mathrm{NO}_{3}\right)_{2(\mathrm{aq}, \mathrm{dil})}$ & $\begin{array}{l}\Delta H_{24}=-2.76 \times(1-M) \pm(-2.76 \times(1-M)) \times \\
((1 / 0.9901-1) / 4)^{-1 *}\end{array}$ \\
\hline $\mathrm{LiOH}_{(\mathrm{aq}, 1 \mathrm{M})}=\mathrm{LiOH}_{(\mathrm{aq}, \mathrm{dil})}$ & $\begin{array}{l}\Delta H_{25}=-1.30 \times(1-M) \pm(-1.30 \times(1-M)) \times \\
((1 / 0.9961-1) / 4)^{-1 *}\end{array}$ \\
\hline
\end{tabular}




\begin{tabular}{|c|c|}
\hline $\mathrm{KOH}_{(\mathrm{aq}, 1 \mathrm{M})}=\mathrm{KOH}_{(\mathrm{aq}, \mathrm{dil})}$ & $\begin{array}{l}\Delta H_{26}=-0.61 \times(1-M) \pm(-0.61 \times(1-M)) \times \\
((1 / 0.9987-1) / 3)^{-1 *}\end{array}$ \\
\hline $\mathrm{CH}_{3} \mathrm{CO}_{2} \mathrm{H}_{(\mathrm{aq}, 1 \mathrm{M})}=\mathrm{CH}_{3} \mathrm{CO}_{2} \mathrm{H}_{(\mathrm{aq}, \text { dil) }}$ & $\begin{array}{l}\Delta H_{27}=0.32 \times(1-M) \pm(0.32 \times(1-M)) \times \\
((1 / 0.9915-1) / 4)^{-1 *}\end{array}$ \\
\hline $\mathrm{UO}_{3(\mathrm{c})}+2 \mathrm{HCl}_{(\mathrm{aq})}=\mathrm{UO}_{2}{ }^{2+}{ }_{(\mathrm{aq})}+2 \mathrm{Cl}_{(\mathrm{aq})}^{-}+\mathrm{H}_{2} \mathrm{O}_{(\mathrm{l})}$ & $\Delta H_{28}=-52.6 \pm 0.1$ \\
\hline $\mathrm{LiOH} \cdot \mathrm{H}_{2} \mathrm{O}_{(\mathrm{c})}+\mathrm{HCl}_{(\mathrm{aq})}=\mathrm{Li}^{+}{ }_{(\mathrm{aq})}+\mathrm{Cl}_{(\mathrm{aq})}^{-}+2 \mathrm{H}_{2} \mathrm{O}_{(\mathrm{l})}$ & $\Delta H_{29}=-58.7 \pm 1.6$ \\
\hline $\mathrm{KOH}_{(\mathrm{c})}+\mathrm{HCl}_{(\mathrm{aq})}=\mathrm{K}_{(\mathrm{aq})}^{+}+\mathrm{Cl}_{(\mathrm{aq})}^{-}+\mathrm{H}_{2} \mathrm{O}_{(\mathrm{l})}$ & $\Delta H_{30}=-56.4 \pm 0.7$ \\
\hline $\mathrm{MoO}_{3(\mathrm{c})}+2 \mathrm{NaOH}_{(\mathrm{aq})}=\mathrm{Na}_{2} \mathrm{MoO}_{4(\mathrm{aq}, \text { dil })}+\mathrm{H}_{2} \mathrm{O}_{(\mathrm{l})}$ & $\Delta H_{31}=-83.3 \pm 2.9$ \\
\hline $\begin{array}{l}\mathrm{UO}_{2}\left(\mathrm{NO}_{3}\right)_{2} \cdot 6 \mathrm{H}_{2} \mathrm{O}_{(\mathrm{c})}+\mathrm{n} \mathrm{H} \mathrm{H}_{2} \mathrm{O}=\mathrm{UO}_{2}\left(\mathrm{NO}_{3}\right)_{2(\mathrm{aq})}+(\mathrm{n}+6) \\
\mathrm{H}_{2} \mathrm{O}_{(\mathrm{l})}\end{array}$ & $\Delta H_{32}=-7.39 \pm 0.50$ \\
\hline $\mathrm{UO}_{3\left(\mathrm{c}, 25^{\circ} \mathrm{C}\right)}=\mathrm{UO}_{3\left(\mathrm{soln}, 700^{\circ} \mathrm{C}\right)}$ & $\Delta H_{33}=\Delta H_{d s}=9.49 \pm 1.53^{5}$ \\
\hline $\mathrm{Li}_{2} \mathrm{O}_{\left(\mathrm{c}, 25^{\circ} \mathrm{C}\right)}=\mathrm{Li}_{2} \mathrm{O}_{\left(\text {soln }, 700^{\circ} \mathrm{C}\right)}$ & $\Delta H_{34}=\Delta H_{d s}=-90.3 \pm 2.57^{6}$ \\
\hline $\mathrm{MoO}_{3\left(\mathrm{c}, 25^{\circ} \mathrm{C}\right)}=\mathrm{MoO}_{3\left(\mathrm{soln}, 700^{\circ} \mathrm{C}\right)}$ & $\Delta H_{35}=\Delta H_{d s}=-72.85 \pm 0.61^{7}$ \\
\hline $\mathrm{Na}_{2} \mathrm{O}_{\left(\mathrm{c}, 25^{\circ} \mathrm{C}\right)}=\mathrm{Na}_{2} \mathrm{O}_{\left(\text {soln }, 700^{\circ} \mathrm{C}\right)}$ & $\Delta H_{36}=\Delta H_{d s}=-217.56 \pm 4.3^{8}$ \\
\hline $\mathrm{H}_{2} \mathrm{O}_{\left(\mathrm{l}, 25^{\circ} \mathrm{C}\right)}=\mathrm{H}_{2} \mathrm{O}_{\left(\mathrm{g}, 700^{\circ} \mathrm{C}\right)}$ & $\Delta H_{37}=69^{9}$ \\
\hline
\end{tabular}




\begin{tabular}{|c|c|}
\hline $\mathrm{O}_{2\left(\mathrm{~g}, 25^{\circ} \mathrm{C}\right)}=\mathrm{O}_{2\left(\mathrm{~g}, 700^{\circ} \mathrm{C}\right)}$ & $\Delta H_{38}=21.74^{9}$ \\
\hline $\mathrm{U}_{(\mathrm{s})}+\mathrm{N}_{2(\mathrm{~g})}+7 \mathrm{O}_{2(\mathrm{~g})}+6 \mathrm{H}_{2(\mathrm{~g})}=\mathrm{UO}_{2}\left(\mathrm{NO}_{3}\right)_{2} \cdot 6 \mathrm{H}_{2} \mathrm{O}_{(\mathrm{c})}$ & $\begin{array}{l}\Delta H_{39}=\Delta H_{\mathrm{f}}^{\circ}\left(\mathrm{UO}_{2}\left(\mathrm{NO}_{3}\right)_{2(\mathrm{c})}\right)=-3,167.5 \pm \\
1.5^{10}\end{array}$ \\
\hline $\mathrm{Li}_{(\mathrm{s})}+1 / 2 \mathrm{O}_{2(\mathrm{~g})}+1 / 2 \mathrm{H}_{2(\mathrm{~g})}=\mathrm{LiOH}_{(\mathrm{aq})}$ & $\Delta H_{40}=\Delta H_{\mathrm{f}}^{\circ}\left(\mathrm{LiOH}_{(\mathrm{aq})}\right)=-508.4 \pm 0.1^{11}$ \\
\hline $\mathrm{K}_{(\mathrm{s})}+1 / 2 \mathrm{O}_{2(\mathrm{~g})}+1 / 2 \mathrm{H}_{2(\mathrm{~g})}=\mathrm{KOH}_{(\mathrm{aq})}$ & $\Delta H_{41}=\Delta H_{f}^{\circ}\left(\mathrm{KOH}_{(\mathrm{aq})}\right)=-482.1 \pm 0.1^{12}$ \\
\hline $2 \mathrm{C}_{(\mathrm{s})}+\mathrm{O}_{2(\mathrm{~g})}+2 \mathrm{H}_{2(\mathrm{~g})}=\mathrm{CH}_{3} \mathrm{CO}_{2} \mathrm{H}_{(\mathrm{aq})}$ & $\begin{array}{l}\Delta H_{42}=\Delta H_{\mathrm{f}}^{\circ}\left(\mathrm{CH}_{3} \mathrm{CO}_{2} \mathrm{H}_{(\mathrm{aq})}\right)=-510.98 \pm \\
0.4^{13}\end{array}$ \\
\hline $\mathrm{Na}(\mathrm{s})+1 / 2 \mathrm{O}_{2(\mathrm{~g})}+1 / 2 \mathrm{H}_{2(\mathrm{~g})}=\mathrm{NaOH}_{(\mathrm{aq})}$ & $\Delta H_{43}=\Delta H_{f}^{\circ}\left(\mathrm{NaOH}_{(\mathrm{aq})}\right)=-470.4 \pm 0.1^{12}$ \\
\hline $\mathrm{U}_{(\mathrm{s})}+1 \frac{1}{2} \mathrm{O}_{2(\mathrm{~g})}=\mathrm{UO}_{3(\mathrm{c})}$ & $\Delta H_{44}=\Delta H_{\mathrm{f}}^{\circ}\left(\mathrm{UO}_{3(\mathrm{~s})}\right)=-1,223.8 \pm 0.8^{10}$ \\
\hline $\mathrm{Li}_{(\mathrm{s})}+\mathrm{O}_{2(\mathrm{~g})}+1 \frac{1}{2} \mathrm{H}_{2(\mathrm{~g})}=\mathrm{LiOH} \cdot \mathrm{H}_{2} \mathrm{O}_{(\mathrm{c})}$ & $\begin{array}{l}\Delta H_{45}=\Delta H_{\mathrm{f}}{ }^{\circ}\left(\mathrm{LiOH} \cdot \mathrm{H}_{2} \mathrm{O}_{(\mathrm{s})}\right)=-787.5 \pm \\
01.6^{11}\end{array}$ \\
\hline $\mathrm{K}_{(\mathrm{s})}+1 / 2 \mathrm{O}_{2(\mathrm{~g})}+1 / 2 \mathrm{H}_{2(\mathrm{~g})}=\mathrm{KOH}_{(\mathrm{c})}$ & $\Delta H_{46}=\Delta H_{f}^{\circ}\left(\mathrm{KOH}_{(\mathrm{s})}\right)=-423.57 \pm 0.14^{14}$ \\
\hline $\mathrm{Mo}_{(\mathrm{s})}+1 \frac{1}{2} \mathrm{O}_{2(\mathrm{~g})}=\mathrm{MoO}_{3(\mathrm{c})}$ & $\Delta H_{47}=\Delta H_{\mathrm{f}}{ }^{\circ}\left(\mathrm{MoO}_{3(\mathrm{~s})}\right)=-745.2 \pm 0.4^{15}$ \\
\hline $\mathrm{H}_{2(\mathrm{~g})}+1 / 2 \mathrm{O}_{2(\mathrm{~g})}=\mathrm{H}_{2} \mathrm{O}_{(\mathrm{l})}$ & $\Delta H_{48}=\Delta H_{\mathrm{f}}^{\circ}\left(\mathrm{H}_{2} \mathrm{O}_{(\mathrm{I})}\right)=-285.8 \pm 0.1^{15}$ \\
\hline $1 / 2 \mathrm{H}_{2(\mathrm{~g})}+1 / 2 \mathrm{Cl}_{2(\mathrm{~g})}=\mathrm{HCl}_{(\mathrm{aq})}$ & $\Delta H_{49}=\Delta H_{\mathrm{f}}^{\circ}\left(\mathrm{HCl}_{(\mathrm{aq})}\right)=-166.266 \pm 0.1^{14}$ \\
\hline
\end{tabular}




\begin{tabular}{|c|c|}
\hline $1 / 2 \mathrm{H}_{2(\mathrm{~g})}+1 / 2 \mathrm{~N}_{2(\mathrm{~g})}+1 \frac{1}{2} \mathrm{O}_{2(\mathrm{~g})}=\mathrm{HNO}_{3(\mathrm{aq})}$ & $\Delta H_{50}=\Delta H_{\mathrm{f}}^{\circ}\left(\mathrm{HNO}_{3(\mathrm{aq})}\right)=-206.85 \pm 0.4^{12}$ \\
\hline $1 / 2 \mathrm{Cl}_{2(\mathrm{~g})}=\mathrm{Cl}_{(\mathrm{aq})}^{-}$ & $\Delta H_{51}=\Delta H_{\mathrm{f}}{ }^{\circ}\left(\mathrm{Cl}^{-}{ }_{(\mathrm{aq})}\right)=-166.266 \pm 0.1^{14}$ \\
\hline $1 / 2 \mathrm{~N}_{2(\mathrm{~g})}+1 \frac{1}{2} \mathrm{O}_{2(\mathrm{~g})}=\mathrm{NO}_{3}^{-}(\mathrm{aq})$ & $\Delta H_{52}=\Delta H_{f^{\circ}}\left(N_{3}{ }_{(a q)}^{-}\right)=-206.85 \pm 0.4^{12}$ \\
\hline $1 / 2 \mathrm{~N}_{2(\mathrm{~g})}+1 \frac{1}{2} \mathrm{H}_{2(\mathrm{~g})}=\mathrm{NH}_{3(\mathrm{~g})}$ & $\Delta H_{53}=\Delta H_{\mathrm{f}}{ }^{\circ}\left(\mathrm{NH}_{3(\mathrm{~g})}\right)=-45.9 \pm 0.4^{16}$ \\
\hline $2 \mathrm{Li}_{(\mathrm{s})}+1 / 2 \mathrm{O}_{2(\mathrm{~g})}=\mathrm{Li}_{2} \mathrm{O}_{(\mathrm{s})}$ & $\Delta H_{54}=\Delta H_{\mathrm{f}}^{\circ}\left(\mathrm{Li}_{2} \mathrm{O}_{(\mathrm{s})}\right)=-597.9 \pm 2.1^{15}$ \\
\hline $2 \mathrm{Na}_{(\mathrm{s})}+1 / 2 \mathrm{O}_{2(\mathrm{~g})}=\mathrm{Na}_{2} \mathrm{O}_{(\mathrm{s})}$ & $\Delta H_{55}=\Delta H_{\mathrm{f}}^{\circ}\left(\mathrm{Na}_{2} \mathrm{O}_{(\mathrm{s})}\right)=-414.82 \pm 0.28^{15}$ \\
\hline $\begin{array}{l}24 \mathrm{Li}_{(\mathrm{s})}+24 \mathrm{U}_{(\mathrm{s})}+60 \mathrm{O}_{2(\mathrm{~g})}+12 \mathrm{H}_{2(\mathrm{~g})}= \\
\mathrm{Li}_{24}\left[\mathrm{UO}_{2} \mathrm{O}_{2} \mathrm{OH}\right]_{24(\mathrm{aq})}\end{array}$ & $\begin{array}{l}\Delta H_{56}=\Delta H_{\mathrm{f}}^{\circ}\left(\mathrm{Li}-\mathrm{U}_{24(\mathrm{aq})}\right)=-\Delta H_{7}+24 \Delta H_{20} \\
+24 \Delta H_{21}+24 \Delta H_{24}+24 \Delta H_{25}+24 \Delta H_{32} \\
24 \Delta H_{39}+24 \Delta H_{40}-120 \Delta H_{48}-24 \Delta H_{49} \\
-24 \Delta H_{50}+24 \Delta H_{51}-24 \Delta H_{52}=- \\
44,377.9 \pm 24.1\end{array}$ \\
\hline $28 \mathrm{Li}_{(\mathrm{s})}+28 \mathrm{U}_{(\mathrm{s})}+70 \mathrm{O}_{2(\mathrm{~g})}=\mathrm{Li}_{28}\left[\mathrm{UO}_{2}\left(\mathrm{O}_{2}\right)_{1.5}\right]_{28(\mathrm{aq})}$ & $\begin{array}{l}\Delta H_{57}=\Delta H_{\mathrm{f}}^{\circ}\left(\mathrm{Li}-\mathrm{U}_{28(\mathrm{aq})}\right)=-\Delta H_{8}+28 \Delta H_{20} \\
+28 \Delta H_{21}+28 \Delta H_{24}+28 \Delta H_{25}+28 \Delta H_{32} \\
+28 \Delta H_{39}+28 \Delta H_{40}-154 \Delta H_{48}-28 \Delta H_{49} \\
-28 \Delta H_{50}+28 \Delta H_{51}-28 \Delta H_{52}=- \\
47,768.8 \pm 16.3\end{array}$ \\
\hline $28 \mathrm{~K}_{(\mathrm{s})}+28 \mathrm{U}_{(\mathrm{s})}+70 \mathrm{O}_{2(\mathrm{~g})}=\mathrm{K}_{28}\left[\mathrm{UO}_{2}\left(\mathrm{O}_{2}\right)_{1.5}\right]_{28(\mathrm{aq})}$ & $\begin{array}{l}\Delta H_{58}=\Delta H_{\mathrm{f}}{ }^{\circ}\left(\mathrm{K}-\mathrm{U}_{28(\mathrm{aq})}\right)=-\Delta H_{9}+28 \Delta H_{20} \\
+28 \Delta H_{22}+28 \Delta H_{24}+28 \Delta H_{26}+28 \Delta H_{32} \\
+28 \Delta H_{39}+28 \Delta H_{41}-154 \Delta H_{48}-28 \Delta H_{49} \\
-28 \Delta H_{50}+28 \Delta H_{51}-28 \Delta H_{52}=- \\
46,132.6 \pm 1.5\end{array}$ \\
\hline $\begin{array}{l}40 \mathrm{Li}_{(\mathrm{s})}+20 \mathrm{~K}_{(\mathrm{s})}+60 \mathrm{U}_{(\mathrm{s})}+150 \mathrm{O}_{2(\mathrm{~g})}+30 \mathrm{H}_{2(\mathrm{~g})}= \\
\mathrm{Li}_{40} \mathrm{~K}_{20}\left[\mathrm{UO}_{2} \mathrm{O}_{2} \mathrm{OH}\right]_{60(\mathrm{aq})}\end{array}$ & $\begin{array}{l}\Delta H_{59}=\Delta H_{\mathrm{f}}^{\circ}\left(\mathrm{U} 60_{(\mathrm{aq})}\right)=-\Delta H_{10}+60 \Delta H_{20} \\
+40 \Delta H_{21}+20 \Delta H_{22}+60 \Delta H_{24}+40 \Delta H_{25} \\
+20 \Delta H_{26}+60 \Delta H_{32}+60 \Delta H_{39}+40 \Delta H_{40} \\
+20 \Delta H_{41}-300 \Delta H_{48}-60 \Delta H_{49}-60 \Delta H_{50} \\
+60 \Delta H_{51}-60 \Delta H_{52}=-111,123.6 \pm 29.6\end{array}$ \\
\hline
\end{tabular}




\begin{tabular}{|c|c|}
\hline $\begin{array}{l}132 \mathrm{Mo}_{(\mathrm{s})}+60 \mathrm{C}_{(\mathrm{s})}+21 \mathrm{~N}_{2(\mathrm{~g})}+252 \mathrm{O}_{2(\mathrm{~g})}+201 \mathrm{H}_{2(\mathrm{~g})}= \\
\left(\mathrm{NH}_{4}\right)_{42}\left[\mathrm{MoVI}_{72} \mathrm{Mo}_{60} \mathrm{O}_{372}\left(\mathrm{CH}_{3} \mathrm{CO}_{2}\right)_{30}\left(\mathrm{H}_{2} \mathrm{O}\right)_{72}\right]_{(\mathrm{aq})}\end{array}$ & $\begin{array}{l}\Delta H_{60}=\Delta H_{\mathrm{f}}^{\circ}\left(\mathrm{Mo}_{132(\mathrm{aq})}\right)=-\Delta H_{11}+132 \\
\Delta H_{31}+30 \Delta H_{23}+30 \Delta H_{27}+132 \Delta H_{47}+ \\
30 \Delta H_{42}+42 \Delta H_{53}+78 \Delta H_{48}= \\
-137,314.4 \pm 649.6\end{array}$ \\
\hline $\begin{array}{l}15 \mathrm{Na}_{(\mathrm{s})} 153 \mathrm{Mo}_{(\mathrm{s})}+264.25 \mathrm{O}_{2(\mathrm{~g})}+76 \mathrm{H}_{2(\mathrm{~g})}= \\
\mathrm{Na}_{15}\left[\mathrm{MoVI}_{126} \mathrm{Mov}_{28} \mathrm{O}_{462} \mathrm{H}_{14}\left(\mathrm{H}_{2} \mathrm{O}\right)_{70}\right]_{0.5} \\
{\left[\mathrm{MoVI}_{124} \mathrm{Mov}_{28} \mathrm{O}_{457} \mathrm{H}_{14}\left(\mathrm{H}_{2} \mathrm{O}\right)_{68}\right]_{0.5(\mathrm{aq})}}\end{array}$ & $\begin{array}{l}4(\mathrm{aq}))=-\Delta H_{12}+153 \\
15 \Delta H_{43}+68.5 \Delta H_{48} \\
1\end{array}$ \\
\hline $\begin{array}{l}24 \mathrm{Li}_{(\mathrm{s})}+24 \mathrm{U}_{(\mathrm{s})}+101.5 \mathrm{O}_{2(\mathrm{~g})}+95 \mathrm{H}_{2(\mathrm{~g})}= \\
\mathrm{Li}_{24}\left[\mathrm{UO}_{2} \mathrm{O}_{2} \mathrm{OH}\right]_{24} \cdot 83 \mathrm{H}_{2} \mathrm{O}_{(\mathrm{c})}\end{array}$ & $\begin{array}{l}+(\mathrm{c})-(\mathrm{C}-80))=-\Delta H_{13}+ \\
+24 \Delta H_{44}+24 \Delta H_{45} \\
4.4 \pm 264.8\end{array}$ \\
\hline $\begin{array}{l}28 \mathrm{Li}_{(\mathrm{s})}+28 \mathrm{U}_{(\mathrm{s})}+106.5 \mathrm{O}_{2(\mathrm{~g})}+73 \mathrm{H}_{2(\mathrm{~g})}= \\
\mathrm{Li}_{28}\left[\mathrm{UO}_{2}\left(\mathrm{O}_{2}\right)_{1.5}\right]_{28} \cdot 73 \mathrm{H}_{2} \mathrm{O}_{(\mathrm{c})}\end{array}$ & $\begin{array}{l}\left.U_{28(\mathrm{c})}\right)=-\Delta H_{14}+28 \Delta H_{28} \\
\Delta H_{44}+28 \Delta H_{45}+31 \Delta H_{48} \\
20.3\end{array}$ \\
\hline $\begin{array}{l}28 \mathrm{~K}_{(\mathrm{s})}+28 \mathrm{U}_{(\mathrm{s})}+91.75 \mathrm{O}_{2(\mathrm{~g})}+43.5 \mathrm{H}_{2(\mathrm{~g})}= \\
\mathrm{K}_{28}\left[\mathrm{UO}_{2}\left(\mathrm{O}_{2}\right)_{1.5}\right]_{28} \cdot 43.5 \mathrm{H}_{2} \mathrm{O}_{(\mathrm{c})}\end{array}$ & $\begin{array}{l})=-\Delta H_{15}+28 \Delta H_{28} \\
H_{44}+28 \Delta H_{46}+29.5 \\
406.9\end{array}$ \\
\hline $\begin{array}{l}\text { s) }+20 \mathrm{~K}_{(\mathrm{s})}+60 \mathrm{U}_{(\mathrm{s})}+263.5 \mathrm{O}_{2(\mathrm{~g})}+257 \mathrm{H}_{2(\mathrm{~g})}= \\
{\left[\mathrm{UO}_{2} \mathrm{O}_{2} \mathrm{OH}\right]_{60} \cdot 227 \mathrm{H}_{2} \mathrm{O}_{(\mathrm{c})}}\end{array}$ & $\begin{array}{l}\Delta H_{65}=\Delta H_{\mathrm{f}}^{\circ}\left(\mathrm{U} 60_{(\mathrm{c})}\right)=-\Delta H_{16}+60 \Delta H_{28}+ \\
40 \Delta H_{29}+20 \Delta H_{30}+60 \Delta H_{44}+40 \Delta H_{45} \\
+20 \Delta H_{46}+187 \Delta H_{48}=-166,809.6 \pm \\
34.1\end{array}$ \\
\hline $\begin{array}{l}132 \mathrm{Mo}_{(\mathrm{s})}+60 \mathrm{C}_{(\mathrm{s})}+21 \mathrm{~N}_{2(\mathrm{~g})}+421.5 \mathrm{O}_{2(\mathrm{~g})}+540 \mathrm{H}_{2(\mathrm{~g})}= \\
\left(\mathrm{NH}_{4}\right)_{42}\left[\mathrm{MoVI}_{72} \mathrm{Mo}_{60} \mathrm{O}_{372}\left(\mathrm{CH}_{3} \mathrm{CO}_{2}\right)_{30}\left(\mathrm{H}_{2} \mathrm{O}\right)_{72}\right] \cdot 339 \mathrm{H}_{2} \mathrm{O}_{(\mathrm{c})}\end{array}$ & $\begin{array}{l}\Delta H_{66}=\Delta H_{\mathrm{f}}^{\circ}\left(\mathrm{Mo}_{132(\mathrm{c})}\right)=-\Delta H_{17}+132 \\
\Delta H_{31}+30 \Delta H_{23}+30 \Delta H_{27}+132 \Delta H_{47}+ \\
30 \Delta H_{42}+42 \Delta H_{53}+417 \Delta H_{48}=- \\
234,520.8 \pm 521.2\end{array}$ \\
\hline $\begin{array}{l}12 \mathrm{Li}_{2} \mathrm{O}_{\left(\mathrm{c}, 25^{\circ} \mathrm{C}\right)}+24 \mathrm{UO}_{3\left(\mathrm{c}, 25^{\circ} \mathrm{C}\right)}+12 \mathrm{O}_{2\left(\mathrm{~g}, 25^{\circ} \mathrm{C}\right)}+95 \mathrm{H}_{2} \mathrm{O}_{(\mathrm{l},} \\
\left.25^{\circ} \mathrm{C}\right)=\mathrm{Li}_{24}\left[\mathrm{UO}_{2} \mathrm{O}_{2} \mathrm{OH}\right]_{24^{\circ}} \cdot 83 \mathrm{H}_{2} \mathrm{O}_{\text {(c) }}\end{array}$ & $\begin{array}{l}\left.J_{24(c)}\right)=-\Delta H_{18}+24 \Delta H_{33} \\
H_{37}+12 \Delta H_{38}=\end{array}$ \\
\hline $\begin{array}{l}7.5 \mathrm{Na}_{2} \mathrm{O}_{\left(\mathrm{c}, 25^{\circ} \mathrm{C}\right)}+153 \mathrm{MoO}_{3\left(\mathrm{c}, 25^{\circ} \mathrm{C}\right)}+460 \mathrm{H}_{2} \mathrm{O}_{\left(\mathrm{l}, 25^{\circ} \mathrm{C}\right)}-7 \\
\mathrm{O}_{2\left(\mathrm{~g}, 25^{\circ} \mathrm{C}\right)}=\mathrm{Na}_{15}\left[\mathrm{Mo}^{\mathrm{VI}}{ }_{126} \mathrm{Mov}_{28} \mathrm{O}_{462} \mathrm{H}_{14}\left(\mathrm{H}_{2} \mathrm{O}\right)_{70}\right]_{0.5} \\
{\left[\mathrm{Movi}_{124} \mathrm{Mov}_{28} \mathrm{O}_{457} \mathrm{H}_{14}\left(\mathrm{H}_{2} \mathrm{O}\right)_{68}\right]_{0.5} \cdot 384 \mathrm{H}_{2} \mathrm{O}_{(\mathrm{c})}}\end{array}$ & $\begin{array}{l}154(\mathrm{c}))=-\Delta H_{19}+7.5 \\
460 \Delta H_{37}-7 \Delta H_{38}=-\end{array}$ \\
\hline $\begin{array}{l}24 \mathrm{Li}_{(\mathrm{s})} 24 \mathrm{U}_{(\mathrm{s})}+101.5 \mathrm{O}_{2(\mathrm{~g})}+95 \mathrm{H}_{2(\mathrm{~g})}= \\
\mathrm{Li}_{24}\left[\mathrm{UO}_{2} \mathrm{O}_{2} \mathrm{OH}\right]_{24} \cdot 83 \mathrm{H}_{2} \mathrm{O}_{(\mathrm{c})}\end{array}$ & 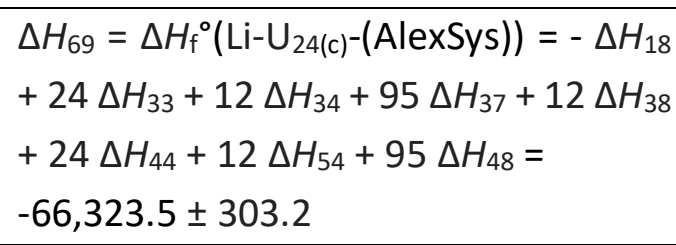 \\
\hline $\begin{array}{l}15 \mathrm{Na}(\mathrm{s})+153 \mathrm{Mo}_{(\mathrm{s})}+456.25 \mathrm{O}_{2(\mathrm{~g})}+460 \mathrm{H}_{2(\mathrm{~g})}= \\
\mathrm{Na}_{15}\left[\mathrm{MoVI}_{126} \mathrm{Mov}_{28} \mathrm{O}_{462} \mathrm{H}_{14}\left(\mathrm{H}_{2} \mathrm{O}\right)_{70}\right]_{0.5} \\
{\left[\mathrm{MoVI}_{124} \mathrm{Mov}_{28} \mathrm{O}_{457} \mathrm{H}_{14}\left(\mathrm{H}_{2} \mathrm{O}\right)_{68}\right]_{0.5} \cdot 384 \mathrm{H}_{2} \mathrm{O}_{(\mathrm{c})}}\end{array}$ & $\begin{array}{l}\Delta H_{70}=\Delta H_{\mathrm{f}}{ }^{\circ}\left(\mathrm{Mo}_{154(\mathrm{c})}\right)=-\Delta H_{19}+7.5 \Delta H_{36} \\
+153 \Delta H_{35}+460 \Delta H_{37}-7 \Delta H_{38}+7.5 \\
\Delta H_{55}+153 \Delta H_{47}+460 \Delta H_{48}= \\
-260,336.9 \pm 4343.1\end{array}$ \\
\hline
\end{tabular}




\section{*The enthalpy value and the error is derived from the thermographs in Section 8 where $M$ is} molarity and error is computed using Equation S3.

$$
\text { Error }=\Delta H \times \sqrt{\frac{1}{\frac{\left(R^{2}\right)^{2}}{4}-1}}
$$

Equation S3

\section{References}

1. Hickam, S.; Aksenov, S. M.; Dembowski, M.; Perry, S. N.; Traustason, H.; Russell, M.; Burns, P. C., Complexity of Uranyl Peroxide Cluster Speciation from Alkali-Directed Oxidative Dissolution of Uranium Dioxide. Inorganic Chemistry 2018, 57 (15), 9296-9305.

2. Sigmon, G. E.; Unruh, D. K.; Ling, J.; Weaver, B.; Ward, M.; Pressprich, L.; Simonetti, A.; Burns, P. C., Symmetry versus Minimal Pentagonal Adjacencies in Uranium-Based Polyoxometalate Fullerene Topologies. Angewandte Chemie-International Edition 2009, 48 (15), 2737-2740.

3. Traustason, H.; Aksenov, S. M.; Burns, P. C., The lithium-water configuration encapsulated by uranyl peroxide cage cluster U-24. Crystengcomm 2019, 21 (3), 390-393.

4. Müller, A.; Das, S.; Krickemeyer, E.; Kuhlmann, C., Polyoxomolybdate clusters: Giant wheels and balls. Inorganic Syntheses 2004, 34, 191-200.

5. Helean, K. B.; Navrotsky, A.; Vance, E. R.; Carter, M. L.; Ebbinghaus, B.; Krikorian, O.; Lian, J.; Wang, L. M.; Catalano, J. G., Enthalpies of formation of Ce-pyrochlore, Ca0.93Ce1.00Ti2.03507.00, U-pyrochlore, Ca1.46U4+0.23U6+0.46Ti1.8507.00 and Gd-pyrochlore, Gd2Ti2O7: three materials relevant to the proposed waste form for excess weapons plutonium. Journal of Nuclear Materials 2002, 303 (2), 226-239.

6. McHale, J. M.; Navrotsky, A.; DiSalvo, F. J., Energetics of ternary nitride formation in the (Li,Ca)-(B,Al)-N system. Chemistry of Materials 1999, 11 (4), 1148-1152.

7. Ranade, M. R.; Elder, S. H.; Navrotsky, A., Energetics of Nanoarchitectured TiO2-ZrO2 and TiO2-MoO3 Composite Materials. Chemistry of Materials 2002, 14 (3), 1107-1114.

8. Kubatko, K. A.; Helean, K. B.; Navrotsky, A.; Burns, P. C., Thermodynamics of uranyl minerals: Enthalpies of formation of rutherfordine, $\mathrm{UO} 2 \mathrm{CO} 3$, andersonite, $\mathrm{Na} 2 \mathrm{CaUO} 2(\mathrm{CO} 3)(3)(\mathrm{H} 2 \mathrm{O})(5)$, and grimselite, $\mathrm{K} 3 \mathrm{NaUO} 2(\mathrm{CO} 3)(3) \mathrm{H} 2 \mathrm{O}$. American Mineralogist 2005, 90 (8-9), 1284-1290.

9. Kubatko, K. A. H.; Helean, K. B.; Navrotsky, A.; Burns, P. C., Stability of peroxide-containing uranyl minerals. Science 2003, 302 (5648), 1191-1193.

10. Grenthe, I.; Fuger, J.; Konings, R. J. M.; Lemire, R. J.; Muller, A. B.; Nguyen-Trung Cregu, C.; Wanner, H., Chemical Thermodynamics of Uranium, 1. Nuclear Energy Agency: North-Holland Amsterdam, 1992.

11. Monnin, C.; Dubois, M., Thermodynamics of the $\mathrm{LiOH}+\mathrm{H} 2 \mathrm{O}$ system. Journal of Chemical and Engineering Data 2005, 50 (4), 1109-1113.

12. Cox, J. D.; Wagman, D. D.; Medvedev, V. A. In CODATA key values for thermodynamics 1989.

13. Hughes, J. T.; Navrotsky, A., Enthalpy of formation of zinc acetate dihydrate. Journal of Chemical Thermodynamics 2011, 43 (6), 980-982.

14. Konings, R. J. M.; Cordfunke, E. H. P.; Ouweltjes, W., THE STANDARD ENTHALPIES OF FORMATION OF HYDROXIDES .2. THE ALKALI HYDROXIDES CSOH AND KOH. Journal of Chemical Thermodynamics 1988, 20 (7), 777-780.

15. Robie, R. A.; Hemingway, B. S., Thermodynamic properties of minerals and related substances at $298.15 \mathrm{~K}$ and 1 bar (10^5 pascals) pressure and at higher temperatures. U.S. Geological Survey 1995, 461.

16. Vanderzee, C. E.; King, D. L., ENTHALPIES OF SOLUTION AND FORMATION OF AMMONIA. Journal of Chemical Thermodynamics 1972, 4 (5), 675-+. 